\title{
Stochastic Optimal Growth with Bounded or Unbounded Utility and with Bounded or Unbounded Shocks
}

\author{
Takashi Kamihigashi* \\ RIEB, Kobe University, 2-1 Rokkodai, Nada, Kobe, 657-8501, Japan \\ CERMSEM, Centre d'Economie de la Sorbonne, 106-112 boulevard de l'Hôpital, 75647 Paris, France \\ E-mail address: tkamihig@rieb.kobe-u.ac.jp
}

May 29, 2006

\begin{abstract}
This paper studies a one-sector stochastic optimal growth model with i.i.d. productivity shocks in which utility is allowed to be bounded or unbounded, the shocks are allowed to be bounded or unbounded, and the production function is not required to satisfy the Inada conditions at zero and infinity. Our main results are threefold. First, we confirm the Euler equation as well as the existence of a continuous optimal policy function under a minimal set of assumptions. Second, we establish the existence of an invariant distribution under quite general assumptions. Third, we show that the output density converges to a unique invariant density independently of initial output under the assumption that the shock distribution has a density whose support is an interval, bounded or unbounded. In addition, we provide existence and stability results for general one-dimensional Markov processes.
\end{abstract}

JEL Classification: C61; C62; E32; O41

Keywords: Stochastic growth; Unbounded utility; Bounded or unbounded shocks; Markov processes; Existence and stability of a invariant distribution

${ }^{*}$ I would like to thank John Stachurski for providing numerous helpful comments as well as for suggesting an extension of Nishimura and Stachurski's (2005) analysis to the case of bounded shocks. Financial support from the 21st Century COE Program at GSE and RIEB, Kobe University, is gratefully acknowledged. 


\section{Introduction}

Recently there has been a resurgence of interest in stochastic growth theory (e.g., Stachurski, 2002; Nishimura and Stachurski, 2005; Kamihigashi, 2005; Mitra et al. 2004; Mitra and Roy, 2006). One of the important recent developments is that the global stability of the optimal output (or capital) process, originally shown by Brock and Mirman (1972) in the case of bounded shocks, has been extended to the case of unbounded shocks by Stachurski (2002), Nishimura and Stachurski (2005), and Zhang (2005). ${ }^{1}$ Many of their arguments, however, rest on the assumption of unbounded shocks, and do not immediately apply to the case of bounded shocks. One of the purposes of this paper is to offer a unified argument that applies simultaneously to both bounded and unbounded cases.

Another purpose of this paper is to confirm the Euler equation as well as the existence of a continuous optimal policy function even when utility is unbounded. While it is extremely common to assume unbounded utility in applications, the Euler equation and the existence of a continuous optimal policy function have been formally verified only in the case of bounded utility except for a few parametric examples. ${ }^{2}$ With bounded utility, standard arguments imply that the value function is the unique solution of the Bellman equation (e.g., Stokey and Lucas, 1989), so that one can immediately derive the Euler equation by applying the envelope theorem to the Bellman equation. While various results are available for cases with unbounded utility concerning the Bellman equation and the existence of an optimal policy function (e.g., Schäl, 1975; Bhattacharaya and Majumdar, 1989; HernándezLerma and Lasserre, 1996, 1999; Durán, 2003), no result seems to be available that allows one to directly obtain the Bellman and Euler equations for the one-sector growth model when utility is unbounded both below and above. ${ }^{3}$

For the above and other purposes, we study a one-sector stochastic growth model with i.i.d. productivity shocks in which utility is allowed to be bounded or unbounded, the shocks are allowed to be bounded or unbounded, and the

\footnotetext{
${ }^{1}$ See Olson and Roy (2005) for a recent survey of the stochastic growth literature.

${ }^{2}$ Most of the literature has relied on Mirman and Zilcha (1975). Their result assumes that utility is bounded below and that consumption is bounded above (rather than utility being bounded above) along any feasible process.

${ }^{3}$ Note that there is no result in Stokey and Lucas (1989, Section 9.1) that shows that the value function solves the Bellman equation. See Le Van and Morhaim (2002) for related results in the deterministic case.
} 
production function is not required to satisfy the Inada conditions at zero and infinity. Our main results are threefold. First, we confirm the Euler equation and the existence of a continuous optimal policy function under a minimal set of assumptions. Second, we establish the existence of an invariant (or stationary) distribution under quite general assumptions. Essentially, the only additional requirement for this result is that the marginal product of capital be not too small when the capital stock is very small, and not too large when the capital stock is very large. Third, we establish the global stability of the optimal output process by showing that the probability density of optimal output converges to a unique invariant density independently of initial output. For this result, we assume that the shock distribution has a density whose support is an interval, bounded or unbounded.

Our existence and stability results are obtained by extending Nishimura and Stachurski's (2005) arguments. Our contribution is that except for concavity of the production function, our assumptions are considerably weaker than those of Stachurski (2002) and Nishimura and Stachurski (2005). In particular we do not assume multiplicative or unbounded shocks; nor do we require the production function to satisfy the Inada condition at infinity. Furthermore our existence result does not require the shock distribution to have a density or the production function to depend positively on the shock. ${ }^{4}$ To our knowledge, our existence result is the most general one currently available on the Brock-Mirman model with i.i.d. shocks. ${ }^{5}$

In addition to our main results, we show existence and stability results for general one-dimensional Markov processes. Though they are not difficult to prove based on well known results on Markov processes, our results are easy to apply since they are stated in terms of primitives, allowing one to bypass numerous definitions required to understand many of the underlying results. Our general results also clarify what needs to be shown for our existence and stability results for the one-sector model.

The rest of the paper is organized as follows. Section 2 presents the model and basic assumptions, and confirms the Euler equation as well as the existence of a continuous optimal policy function. Section 3 establishes

\footnotetext{
${ }^{4}$ Mirman and Zilcha (1975) treated such a case with bounded shocks.

${ }^{5}$ Our existence result is also weaker than that of Zhang (2005), which assumes that utility is bounded below and the production function satisfies both Inada conditions. Our and his stability results cannot be ranked since his result assumes unbounded shocks but, like many stability results in the literature, does not require the shock distribution to have a density.
} 
the existence of an invariant distribution after stating an existence result for general one-dimensional Markov processes. Section 4 establishes the global stability of the optimal output process after stating a stability result for general one-dimensional Markov processes. Section 5 concludes the paper by commenting on possible extensions. All proofs are relegated to the appendices.

\section{The Model}

Consider the following maximization problem:

$$
\begin{aligned}
\max _{\left\{c_{t}, k_{t+1}\right\}_{t=0}^{\infty}} & E \sum_{t=0}^{\infty} \beta^{t} u\left(c_{t}\right) \\
\text { s.t. } & \forall t \in \mathbb{Z}_{+}, \quad c_{t}+k_{t+1}=y_{t}, \\
& c_{t}, k_{t+1} \geq 0, \\
& y_{t+1}=f\left(k_{t+1}, z_{t+1}\right), \\
& y_{0} \geq 0 \text { given, }
\end{aligned}
$$

where $\beta \in(0,1)$ is the discount factor, $u$ is the utility function, $f$ is the production function, $c_{t}$ is consumption in period $t, k_{t}$ is the capital stock at the beginning of period $t$, and $z_{t}$ is the productivity shock in period $t$. The infinite sum in (2.1) is understood as a Lebesgue integral, and initial output $y_{0}$ is assumed to be non-random. Let us state and discuss our assumptions.

Assumption 2.1. $u: \mathbb{R}_{+} \rightarrow[-\infty, \infty)$ is $C^{1}$ on $\mathbb{R}_{++}$, continuous, strictly increasing, and strictly concave, satisfying

$$
\lim _{c \downarrow 0} u^{\prime}(c)=\infty .
$$

Note that the utility function $u$ is allowed to be unbounded both above and below.

Assumption 2.2. $f: \mathbb{R}_{+} \times \mathbb{R}_{+} \rightarrow \mathbb{R}_{+}$satisfies the following. (i) $\forall z \geq$ $0, f(0, z)=0$, and $f(\cdot, z): \mathbb{R}_{+} \rightarrow \mathbb{R}_{+}$is $C^{1}$ on $\mathbb{R}_{++}$, continuous, concave, and increasing. ${ }^{6}$ (ii) $\forall x \geq 0, f(x, \cdot): \mathbb{R}_{+} \rightarrow \mathbb{R}_{+}$is a Borel(-measurable) function. ${ }^{7}$

\footnotetext{
${ }^{6}$ In this paper, "increasing" means "nondecreasing." Similar remarks apply to "decreasing," "positive," and "negative."

${ }^{7}$ By Stokey and Lucas (1989, Theorem 7.4), this implies that $f_{1}(x, \cdot)$ is a Borel function since it is the pointwise limit of the sequence $\{[f(x+(1 / n), \cdot)-f(x, \cdot)] n\}_{n=1}^{\infty}$.
} 
The domain of $f$ can alternatively be specified to be the product of $\mathbb{R}_{+}$ and the range of $z_{t}$; in that case, $f$ can easily be extended to $\mathbb{R}_{+}^{2}$ as long as the range of $z_{t}$ belongs to $\mathbb{R}_{+}$. Note that the production function $f$ is not required to satisfy the Inada conditions at zero and infinity; nor is it required to be increasing in the second argument.

Assumption 2.3. $\left\{z_{t}\right\}_{t=1}^{\infty}$ is a positive i.i.d. process with a distribution $\Phi$ : $\mathbb{R}_{+} \rightarrow[0,1]$ such that

$$
\exists x>0, \quad E f_{1}(x, z)>0,
$$

where $z$ is a random variable with the distribution $\Phi .^{8}$

If $E f_{1}(x, z)=0$ for all $x>0$, then $f(x, z)=0$ with probability 1 for all $x>0$. The role of (2.7) is to rule out this trivial case.

We use the following definitions. Given $y_{0} \geq 0$, a stochastic process $\left\{c_{t}, k_{t+1}, y_{t+1}\right\}_{t=0}^{\infty}$ in $\mathbb{R}_{+}^{3}$ is feasible (from $y_{0}$ ) if it satisfies (2.2)-(2.4) and if $\left\{c_{t}\right\}$ is adapted to the filtration generated by $\left\{z_{t}\right\}$. A feasible process is optimal (from $y_{0}$ ) if it solves the maximization problem (2.1)-(2.5). A function $\pi: \mathbb{R}_{+} \rightarrow \mathbb{R}_{+}$is a feasible policy function if for each $y_{0} \geq 0$, the process $\left\{c_{t}, k_{t+1}, y_{t+1}\right\}$ given below is feasible:

$$
\forall t \in \mathbb{Z}_{+}, \quad k_{t+1}=\pi\left(y_{t}\right), c_{t}=y_{t}-\pi\left(y_{t}\right), y_{t+1}=f\left(k_{t+1}, z_{t+1}\right) .
$$

An optimal policy function is a feasible policy function $\pi$ such that for each $y_{0} \geq 0$, the process $\left\{c_{t}, k_{t+1}, y_{t+1}\right\}$ given by (2.8) is optimal. If $\left\{c_{t}, k_{t+1}, y_{t+1}\right\}$ is feasible or optimal, then $\left\{c_{t}\right\},\left\{y_{t}\right\}$, and $\left\{c_{t}, k_{t+1}\right\}$ are called feasible or optimal accordingly.

For $y \in \mathbb{R}_{+}$, define $\left\{\bar{c}_{t}(y)\right\}_{t=0}^{\infty}$ by

$$
\bar{c}_{0}(y)=y, \quad \forall t \in \mathbb{Z}_{+}, \bar{c}_{t+1}(y)=f\left(\bar{c}_{t}(y), z_{t+1}\right) .
$$

Note that $\bar{c}_{t}(y)$ is the largest possible consumption level in period $t$ from initial output $y$.

Assumption 2.4. $\forall y \geq 0, E \sum_{t=0}^{\infty} \beta^{t} u\left(\bar{c}_{t}(y)\right)_{+}<\infty .^{9}$

\footnotetext{
${ }^{8}$ Unless otherwise indicated, the variable $z$ is understood this way for the rest of the paper.

${ }^{9} u\left(\bar{c}_{t}(y)\right)_{+} \equiv \max \left\{u\left(\bar{c}_{t}(y)\right), 0\right\}$.
} 
This assumption ensures that the objective function is always well defined. It also allows one to apply Tonelli's theorem (e.g., Dudley, 2002, p. 137) to $\beta^{t}\left[u\left(c_{t}\right)-u\left(\bar{c}_{t}(y)\right)_{+}\right] \leq 0$ to obtain

$$
E \sum_{t=0}^{\infty} \beta^{t} u\left(c_{t}\right)=\sum_{t=0}^{\infty} \beta^{t} E u\left(c_{t}\right)
$$

for any feasible consumption process $\left\{c_{t}\right\}$ from $y \geq 0$.

For $y \geq 0$, define

$$
v(y)=\sup E \sum_{t=0}^{\infty} \beta^{t} u\left(c_{t}\right),
$$

where the supremum is taken over all feasible consumption processes $\left\{c_{t}\right\}$ from $y$.

Assumption 2.5. $\forall y>0, v(y)>-\infty$.

This is a minimum requirement for the maximization problem to be nontrivial except when initial output is zero. The assumption simply means that there is at least one feasible process that results in a finite value of the objective function as long as initial output is strictly positive.

The following result confirms, among other things, the Bellman equation, the existence of a unique optimal policy function, and the Euler equation.

Theorem 2.1. Let Assumptions 2.1-2.5 hold. Then $v$ is continuously differentiable on $\mathbb{R}_{++}$, strictly increasing, and strictly concave, satisfying

$$
\forall y \geq 0, \quad v(y)=\max _{0 \leq x \leq y}\{u(y-x)+\beta E v(f(x, z))\} .
$$

Furthermore, there exists a unique optimal policy function $k: \mathbb{R}_{+} \rightarrow \mathbb{R}_{+}$, which satisfies

$$
\forall y>0, \quad 0<k(y)<y
$$

Define $c: \mathbb{R}_{+} \rightarrow \mathbb{R}_{+}$by

$$
\forall y \geq 0, \quad c(y)=y-k(y) .
$$

Then $k$ and $c$ are continuous and strictly increasing, satisfying

$$
\forall y>0, \quad u^{\prime}(c(y))=E\left\{u^{\prime}(c(f(k(y), z))) f_{1}(k(y), z)\right\} .
$$

Proof. See Appendix A. ${ }^{10}$

\footnotetext{
${ }^{10}$ For the rest of the paper including the appendices, we omit expressions like "almost surely" and "almost everywhere" when there is no risk of confusion.
} 


\section{Existence of an Invariant Distribution}

This section offers sufficient conditions for existence of an invariant distribution for the optimal output process. Let us begin by defining the Markov process that the optimal output process $\left\{y_{t}\right\}$ follows. For the rest of the paper, $\left\{y_{t}\right\}$ is understood as the optimal output process unless otherwise indicated.

For $y, z \geq 0$, define

$$
m(y, z)=f(k(y), z) .
$$

Then the output process $\left\{y_{t}\right\}$ follows

$$
\forall t \in \mathbb{Z}_{+}, \quad y_{t+1}=m\left(y_{t}, z_{t+1}\right) .
$$

Let $P(A)$ denote the probability of event $A$. For $y, y^{\prime} \geq 0$, define

$$
Q\left(y, y^{\prime}\right)=P\left(m(y, z) \leq y^{\prime}\right)
$$

which is the conditional distribution of next period output $y^{\prime}$ given current output $y$. For $t \in \mathbb{Z}_{+}$, let $G_{t}$ be the distribution of $y_{t}$. Since $y_{0}$ is non-random, $G_{0}$ assigns all probability mass to $y_{0}$, and $G_{1}$ is simply the conditional distribution of $y^{\prime}$ given $y=y_{0}$ :

$$
\forall y^{\prime} \geq 0, \quad G_{1}\left(y^{\prime}\right)=Q\left(y_{0}, y^{\prime}\right) .
$$

In period $t \in \mathbb{N}, y_{t}$ is distributed as $G_{t}$, so $G_{t+1}$ is the conditional distribution of $y^{\prime}$ given $y$ weighted according to $G_{t}:^{11}$

$$
\forall t \in \mathbb{N}, \forall y^{\prime} \geq 0, \quad G_{t+1}\left(y^{\prime}\right)=\int Q\left(y, y^{\prime}\right) d G_{t}(y) .
$$

An invariant distribution is defined as a distribution $G^{*}: \mathbb{R}_{+} \rightarrow[0,1]$ satisfying

$$
\begin{gathered}
\forall y^{\prime} \geq 0, \quad G^{*}\left(y^{\prime}\right)=\int Q\left(y, y^{\prime}\right) d G^{*}(y), \\
G^{*}(0)=0 .
\end{gathered}
$$

\footnotetext{
${ }^{11}$ Equation (3.5) is a version of the Chapman-Kolmogorov equation (e.g., Meyn and Tweedie, 1996, (3.25)). An easy way to see (3.5) is to note that $G_{t+1}\left(y^{\prime}\right)=P\left(m\left(y_{t}, z_{t+1}\right) \leq\right.$ $\left.y^{\prime}\right)=E 1\left\{m\left(y_{t}, z_{t+1}\right) \leq y^{\prime}\right\}=E_{y_{t}} E_{z_{t+1}} 1\left\{m\left(y_{t}, z_{t+1}\right) \leq y^{\prime}\right\}=E_{y_{t}} Q\left(y_{t}, y^{\prime}\right)$, where $1\{\cdot\}$ is the indicator function and $E_{y_{t}}$ is the expectation with respect to $y_{t}$, etc.
} 
By requiring (3.7), we rule out the trivial distribution that assigns all probability mass to 0 . Note that (3.6) trivially holds if $G^{*}(0)=1$. If there is a distribution $G^{*}$ with $0<G^{*}(0)<1$ that satisfies (3.6), then $G \equiv\left(G^{*}-\right.$ $\left.G^{*}(0)\right) /\left(1-G^{*}(0)\right)$ satisfies (3.6) and (3.7). Hence (3.7) is a normalization condition once the trivial distribution is ruled out.

Let us introduce some assumptions on the production function to ensure the existence of an invariant distribution (which is by definition nontrivial).

Assumption 3.1. $f: \mathbb{R}_{+} \times \mathbb{R}_{+} \rightarrow \mathbb{R}_{+}$is continuous.

This assumption ensures that $m$ as defined in (3.1) is continuous since $k$ is continuous by Theorem 2.1 .

Assumption 3.2. $\exists \hat{x}>0, E f(\hat{x}, z)<\infty$.

This assumption reduces to $E z<\infty$ if

$$
f(x, z)=\hat{f}(x) z+(1-\delta) x
$$

for some $\hat{f}: \mathbb{R}_{+} \rightarrow \mathbb{R}_{+}$and $\delta \in(0,1]$ for all $x \geq 0$. Assumption 3.2 in fact implies

$$
\forall x \geq 0, \quad E f(x, z)<\infty,
$$

since $f(x, z) \leq f(\hat{x}, z)$ for $x \in(0, \hat{x})$, and $f(x, z) / x \leq f(\hat{x}, z) / \hat{x}$ for $x>\hat{x}$ by concavity.

Assumption 3.3. $\lim _{x \uparrow \infty} E f_{1}(x, z)<1$.

Under (3.8) this assumption holds for any $\delta \in(0,1]$ as long as $\lim _{x \uparrow \infty} \hat{f}^{\prime}(x)=$ 0 . By contrast the Inada condition $\lim _{x \uparrow \infty} E f_{1}(x, z)=0$, a typical assumption in the literature, requires $\delta=1$ under (3.8).

The role of Assumptions 3.2 and 3.3 is to prevent probability mass from escaping to infinity. The next two assumptions enable one to prevent probability mass from escaping to zero.

Assumption 3.4. $\exists \tilde{r}, \tilde{x}>0, E f_{1}(\tilde{x}, z)^{-\tilde{r}}<\infty$.

This assumption reduces to $E z^{-\tilde{r}}<\infty$ under (3.8) with $\delta=1$. Note that Assumption 3.4 implies $f_{1}(\tilde{x}, z)>0$ with probability 1 , which in turn implies

$$
\forall x>0, \quad P(f(x, z)>0)=1 .
$$

Since $k(y)>0$ for $y>0$, it follows that

$$
y_{0}>0 \Rightarrow \forall t \in \mathbb{N}, P\left(y_{t}>0\right)=1 \text {. }
$$

Hence we may restrict the state space of $\left\{y_{t}\right\}$ to $\mathbb{R}_{++}$provided $y_{0}>0$. 
Assumption 3.5. $\lim _{x \downarrow 0} E \ln \left[\beta f_{1}(x, z)\right]>0$.

The above expectation is well defined for $x \in(0, \tilde{x}]$ under Assumption 3.4 (by Lemma B.1 with $\zeta=\left\{\beta f_{1}(x, z)\right\}^{-1}$ ). Nishimura and Stachurski (2005) required (3.8) with $\delta=1$ and Assumption 3.4 with $\tilde{r}=1$. They also assumed the following in our notation:

$$
E\left[\frac{1}{\beta f_{1}(0, z)}\right]<1
$$

where $f_{1}(0, z)=\lim _{x \downarrow 0} f_{1}(x, z)$. Assumption 3.5 is strictly weaker than (3.12) as long as $P\left(f_{1}(0, z)<\infty\right)>0$ and $f_{1}(0, z)$ is not a degenerate random variable where $f_{1}(0, z)<\infty$. For then, by Jensen's inequality,

$$
\ln E \frac{1}{\beta f_{1}(0, z)}>E \ln \frac{1}{\beta f_{1}(0, z)}=-E \ln \left[\beta f_{1}(0, z)\right] .
$$

The expectation on the rightmost side equals the limit in Assumption 3.5 by the monotone convergence theorem. We argue below that Assumption 3.5 is possibly the tightest condition on $f$ and $\beta$ to prevent probability mass from escaping to zero.

Following Nishimura and Stachurski (2005), we establish the existence of an invariant distribution by constructing a "Lyapunov" function. The following result gives sufficient conditions for existence of an invariant distribution for general Markov processes on $\mathbb{R}_{+}$.

Lemma 3.1. Let $m: \mathbb{R}_{+} \times \mathbb{R}_{+} \rightarrow \mathbb{R}_{+}$be a continuous function. Let $\left\{z_{t}\right\}$ be a positive i.i.d. process. Consider the Markov process (3.2). Suppose there exists a function $V: \mathbb{R}_{+} \rightarrow[0, \infty]$ such that

$$
\begin{array}{r}
\text { (i) } V(0)=\infty, \quad \text { (ii) } \forall y>0, V(y)<\infty, \\
\text { (i) } \lim _{y \downarrow 0} V(y)=\infty, \quad \text { (ii) } \lim _{y \uparrow \infty} V(y)=\infty, \\
\exists \lambda \in[0,1), \exists \alpha \geq 0, \forall y>0, \quad E[V(m(y, z))] \leq \lambda V(y)+\alpha .
\end{array}
$$

Then there exists an invariant distribution (which is nontrivial by our definition).

Proof. See Appendix B. 
The idea of this result is that an invariant distribution exists as long as probability mass does not escape to zero or infinity. As the proof shows, (3.16) implies that $\left\{E\left[V\left(y_{t}\right)\right]\right\}_{t=0}^{\infty}$ is a bounded sequence. This means that, loosely speaking, $y_{t}$ cannot go to zero since otherwise $E\left[V\left(y_{t}\right)\right]$ blows up by (3.15)(i). Likewise $y_{t}$ cannot go to infinity since otherwise $E\left[V\left(y_{t}\right)\right]$ blows up by (3.15)(ii). As this discussion suggests, the two conditions in (3.15) can be used independently to prevent probability mass from escaping to zero or to infinity. ${ }^{12}$ We are now ready to state the main result of this section.

Theorem 3.1. Under Assumptions 2.1-2.5 and 3.1-3.5, there exists an invariant distribution.

Proof. See Appendix B.

The crucial assumption for preventing probability mass from escaping to zero is Assumption 3.5. If $z$ is non-random, the assumption reduces to $\beta f_{1}(0, z)>1$, which is the well known condition to prevent convergence to zero in the deterministic case. To see that Assumption 3.5 is quite tight even in the stochastic case, let $\theta>0$, and suppose (i) $u(c)=\ln c$, (ii) $f(x, z)=\theta z x$, (iii) $E z^{-\tilde{r}}<\infty$ for some $\tilde{r}>0$, (iv) $E z=1$, and (v) $E|\ln z|<\infty$. In this case it is well known that the optimal policy function is given by $k(y)=\beta y$.

By (iii), Assumption 3.4 holds. Assumption 3.5 now reduces to

$$
E \ln z+\ln (\beta \theta)>0 .
$$

This condition prevents probability mass from escaping to zero independently of Assumptions 3.2 and 3.3. On the other hand, it follows from Kamihigashi (2005, Theorem 3.1) that the output process $\left\{y_{t}\right\}$ from any $y_{0}>0$ converges to zero with probability 1 if $^{13}$

$$
E \ln z+\ln (\beta \theta)<0 .
$$

Since almost sure convergence implies convergence in probability (e.g., Dudley, 2002, p. 261), (3.18) implies that there is no (nontrivial) invariant distribution. In view of (3.17) and (3.18), it seems possible that Assumption 3.5 is

\footnotetext{
${ }^{12}$ See Nishimura and Stachurski (2005, p. 109) for a more formal discussion.

${ }^{13}$ To apply the theorem, in Section 3 of Kamihigashi (2005), assume $k_{t+1}=\beta \theta z_{t} k_{t}$ (which is the optimal capital process) and $c_{t}=0$ for all $t \in \mathbb{Z}_{+}$. See Nishimura et al. (2006) and Mitra and Roy (2005) for related results.
} 
the tightest condition on $f$ and $\beta$ to prevent probability mass from escaping to zero.

By contrast, Assumption 3.3, the main assumption used to prevent probability mass from escaping to infinity, is not as tight as Assumption 3.5. In fact, Assumptions 3.2 and 3.3 can be weakened along the lines of Assumptions 3.4 and 3.5 using Lemma B.1. The weaker versions of these assumptions are not stated here since the original assumptions are easier to interpret and already sufficient for most purposes. In addition, even the weaker version of Assumption 3.3 does not reduce to the corresponding condition in the deterministic case, i.e., $\lim _{x \uparrow \infty} \beta f_{1}(x, z)<1$ with $z$ being non-random. ${ }^{14}$ This can be seen from the fact that Assumption 3.3 does not involve $\beta$. Hence there seems to be room for improving Assumption 3.3 by utilizing the Euler equation or other optimality conditions, though it does not seem trivial.

\section{Global Stability}

This section offers sufficient conditions for global stability of the output process. Let us begin by introducing an assumption on the distribution $\Phi$ of $z_{t}$.

Assumption 4.1. The distribution $\Phi$ has a density $\phi$ such that for some $\underline{z}, \bar{z} \in[0, \infty]$ with $\underline{z}<\bar{z}$, (i) $\forall z \in Z \equiv(\underline{z}, \bar{z}), \phi(z)>0$, (ii) $\forall z \in \mathbb{R}_{+} \backslash$ $[\underline{z}, \bar{z}], \phi(z)=0$, and (iii) there exists a continuous function $\phi: \mathbb{R}_{++} \rightarrow \mathbb{R}_{+}$ such that $\forall z>0, \phi(z) \leq \phi(z)$ and $\forall z \in Z, \phi(z)>0$.

The support of the density $\phi$ is an interval, bounded or unbounded. Beyond this, the only requirement is that $\phi$ be everywhere greater than or equal to some continuous function with essentially the same support. Note that $\phi$ is not required to be continuous or bounded.

Given Assumption 4.1, the following assumption allows us to express the distribution of $y_{t}$ for $t \geq 1$ by a density.

Assumption 4.2. $f$ is $C^{1}$ on $\mathbb{R}_{++} \times \mathbb{R}_{++}$with $f_{2}>0$ on $\mathbb{R}_{++} \times \mathbb{R}_{++}$. If $\bar{z}=\infty$, then $\forall x>0, f(x, \infty) \equiv \lim _{z \uparrow \infty} f(x, z)=\infty$.

Let us show that the distribution of $y_{t}$ for $t \geq 1$ has a density, and derive an equation that characterizes its evolution. First define $\underline{h}:[0, \infty) \rightarrow[0, \infty)$

\footnotetext{
${ }^{14}$ See Kamihigashi and Roy (2005) for such conditions in the deterministic case.
} 
and $\bar{h}:(0, \infty) \rightarrow(0, \infty]$ by

$$
\underline{h}(y)=f(k(y), \underline{z}), \quad \bar{h}(y)=f(k(y), \bar{z}) .
$$

Note that $\underline{h}(y)$ and $\bar{h}(y)$ are the lowest and highest levels of output that can be reached next period from current output $y$. These functions have often been used in the case of bounded shocks (e.g., Brock and Mirman, 1972; Olson and Roy, 2005).

For $y, y^{\prime}>0$ with $y^{\prime} \in(\underline{h}(y), \bar{h}(y))$, define $\zeta\left(y, y^{\prime}\right)$ by

$$
y^{\prime}=f\left(k(y), \zeta\left(y, y^{\prime}\right)\right)
$$

Note that $\zeta\left(y, y^{\prime}\right)$ is continuous in $\left(y, y^{\prime}\right)$, decreasing in $y$, and continuously differentiable in $y^{\prime}$ with

$$
\zeta_{2}\left(y, y^{\prime}\right)=\frac{1}{f_{2}\left(k(y), \zeta\left(y, y^{\prime}\right)\right)} .
$$

Let $y>0$ and $y^{\prime} \in(\underline{h}(y), \bar{h}(y))$. Since $f(x, \cdot)$ is increasing by Assumption 4.2 ,

$$
P\left(f(k(y), z) \leq y^{\prime}\right)=P\left(z \leq \zeta\left(y, y^{\prime}\right)\right)=\Phi\left(\zeta\left(y, y^{\prime}\right)\right) .
$$

Since $Q\left(y, y^{\prime}\right)=P\left(f(k(y), z) \leq y^{\prime}\right)$ by definition (recall (3.3)),

$$
\begin{aligned}
Q\left(y, y^{\prime}\right) & =\Phi\left(\zeta\left(y, y^{\prime}\right)\right) \\
& =\int_{\underline{z}}^{\zeta\left(y, y^{\prime}\right)} \phi(\xi) d \xi \\
& =\int_{\underline{h}(y)}^{y^{\prime}} \phi(\zeta(y, x)) \zeta_{2}(y, x) d x,
\end{aligned}
$$

where the last equality uses the change of variables $\xi=\zeta(y, x)$. Recalling (4.3), we see that the conditional distribution of $y^{\prime}$ given $y$ has density

$$
q\left(y, y^{\prime}\right)= \begin{cases}\frac{\phi\left(\zeta\left(y, y^{\prime}\right)\right)}{f_{2}\left(k(y), \zeta\left(y, y^{\prime}\right)\right)} & \text { if } y^{\prime} \in(\underline{h}(y), \bar{h}(y)) \\ 0 & \text { otherwise. }\end{cases}
$$

This density, like all other probability densities, is unique up to sets of measure zero. It follows from (4.5)-(4.8) that

$$
\forall y, y^{\prime}>0, \quad Q\left(y, y^{\prime}\right)=\int_{0}^{y^{\prime}} q(y, x) d x .
$$


Now by (3.5), (4.9), and Tonelli's theorem, for $y^{\prime}>0$,

$$
\begin{aligned}
G_{t+1}\left(y^{\prime}\right) & =\iint_{0}^{y^{\prime}} q(y, x) d x d G_{t}(y) \\
& =\int_{0}^{y^{\prime}} \int q(y, x) d G_{t}(y) d x .
\end{aligned}
$$

Hence $G_{t+1}$ has density $\int q(y, x) d G_{t}(y)$ whether or not $G_{t}$ has a density. This implies that $G_{1}$ has a density, and any invariant distribution has a density. Thus we restrict attention to densities in what follows.

Let $D$ be the set of probability densities on $\mathbb{R}_{++}$, i.e., the set of Borel functions $g: \mathbb{R}_{++} \rightarrow \mathbb{R}_{+}$such that $\int g(x) d x=1$. We consider densities on $\mathbb{R}_{++}$rather than $\mathbb{R}_{+}$because of (3.11). Given $y_{0}>0$, let $g_{t}^{y_{0}} \in D$ be the density of $y_{t}\left(\right.$ or $\left.G_{t}\right)$ for $t \in \mathbb{N}$. From (4.10) and (4.11),

$$
\forall t \in \mathbb{N}, \forall y^{\prime}>0, \quad g_{t+1}^{y_{0}}\left(y^{\prime}\right)=\int q\left(y, y^{\prime}\right) g_{t}^{y_{0}}(y) d y
$$

Define $T: D \rightarrow D$ by

$$
\forall y^{\prime}>0, \quad(T g)\left(y^{\prime}\right)=\int q\left(y, y^{\prime}\right) g(y) d y
$$

An invariant density is defined as a density $g^{*} \in D$ satisfying $T g^{*}=g^{*}$, i.e.,

$$
\forall y^{\prime}>0, \quad g^{*}\left(y^{\prime}\right)=\int q\left(y, y^{\prime}\right) g^{*}(y) d y
$$

For any function $g: \mathbb{R}_{++} \rightarrow \mathbb{R}_{+}$, define the support of $g$ as

$$
S(g)=\{y>0 \mid g(y)>0\} .
$$

Let $\mathcal{B}$ be the set of Borel subsets of $\mathbb{R}_{++}$. The following result gives sufficient conditions for global stability for general Markov processes on $\mathbb{R}_{++}$.

Lemma 4.1. Let $m: \mathbb{R}_{++}^{2} \rightarrow \mathbb{R}_{++}$. Let $\left\{z_{t}\right\}$ be an i.i.d. process on $\mathbb{R}_{++}$. Consider the Markov process (3.2). Define $Q: \mathbb{R}_{++}^{2} \rightarrow \mathbb{R}_{+}$by (3.3). ${ }^{15}$ Assume the following.

\footnotetext{
${ }^{15}$ Since $Q$ fully characterizes the Markov process, no direct assumptions are needed on $m$ and $\left\{z_{t}\right\}$ for this lemma.
} 
(i) There is a function $V: \mathbb{R}_{++} \rightarrow \mathbb{R}_{+}$satisfying (3.15) and (3.16).

(ii) There is a Borel function $q: \mathbb{R}_{++}^{2} \rightarrow \mathbb{R}_{+}$satisfying (4.9).

(iii) There is a lower semicontinuous function $\underline{q}: \mathbb{R}_{++}^{2} \rightarrow \mathbb{R}_{+}$such that

$$
\begin{aligned}
\forall y, y^{\prime} & >0, \quad \underline{q}\left(y, y^{\prime}\right) \leq q\left(y, y^{\prime}\right), \\
\forall y & >0, \quad \int \underline{q}\left(y, y^{\prime}\right) d y^{\prime}>0, \\
\exists \tilde{y} & >0, \quad \underline{q}(\tilde{y}, \tilde{y})>0 .
\end{aligned}
$$

(iv) There is a set $A \in \mathcal{B}$ of strictly positive Lebesgue measure such that

$$
\forall y_{0}>0, \exists t \in \mathbb{N}, \quad A \subset S\left(g_{t}^{y_{0}}\right)
$$

where $g_{t}^{y_{0}}$ is as defined above. ${ }^{16}$

Then there exists a unique invariant density $g^{*} \in D$. Furthermore, there exist $\rho \in(0,1)$ and $R>0$ such that

$$
\forall y_{0}>0, \forall t \in \mathbb{N}, \quad \int\left|g_{t}^{y_{0}}(y)-g^{*}(y)\right| \tilde{V}(y) d y \leq \rho^{t} R \tilde{V}\left(y_{0}\right),
$$

where $\tilde{V}=V+1$.

Proof. See Appendix C.

We are now ready to state the main result of this section.

Theorem 4.1. Let Assumptions 2.1-2.5, 3.1-3.5, 4.1, and 4.2 hold. Then (i) there exists a function $V: \mathbb{R}_{++} \rightarrow \mathbb{R}_{+}$satisfying (3.15) and (3.16):17 (ii) there exists a unique invariant density $g^{*} \in D$; and (iii) there exist $\rho \in(0,1)$ and $R>0$ satisfying (4.20).

Proof. See Appendix C.

Note that (4.20) implies

$$
\forall y_{0}>0, \forall t \in \mathbb{N}, \quad \int\left|g_{t}^{y_{0}}(y)-g^{*}(y)\right| d y \leq \rho^{t} R \tilde{V}\left(y_{0}\right)
$$

\footnotetext{
${ }^{16}$ Under (4.9), the left-hand side of (4.10) equals the right-hand side of (4.11), so the distribution of $y_{t}$ has a density for $t \geq 1$.

${ }^{17}$ The function $V$ is given by (B.29) and (B.11).
} 
This is the main conclusion of Nishimura and Stachurski (2005, Theorem 3.1). ${ }^{18}$ The additional implication of (4.20) is that the tails of $g_{t}^{y_{0}}$ converge much faster than the middle part by (3.15).

The convergence property (4.20) is known as $V$-uniform ergodicity in the literature on Markov processes (e.g., Meyn and Tweedie, 1996) since (4.20) means that convergence occurs uniformly at a common rate for all $y_{0} \in \mathbb{R}_{++}$ if the distance between $g_{t}^{y_{0}}$ and $g^{*}$ is adjusted appropriately by $V$. In addition to global stability, $V$-uniform ergodicity has various useful implications, as shown by Nishimura and Stachurski (2005).

As mentioned in the introduction, except for concavity of the production function, all our assumptions are weaker than those of Stachurski (2002) and Nishimura and Stachurski (2005). In particular they assumed that $\underline{z}=0$, $\bar{z}=\infty$, and $\phi$ is continuous, in addition to requiring stronger conditions than the assumptions stated in Section 3, as discussed there. Theorem 4.1 confirms their claim that the assumption of unbounded shocks "is not related to the basic idea" of their analysis.

\section{Concluding Remarks}

In this paper we have studied a one-sector stochastic growth model with i.i.d. productivity shocks in which utility is allowed to be bounded or unbounded, the shocks are allowed to be bounded or unbounded, and the production function is not required to satisfy the Inada conditions at zero and infinity. We have (i) confirmed the Euler equation as well as the existence of a continuous optimal policy function under a minimal set of assumptions, (ii) established the existence of an invariant distribution under quite general assumptions, and (iii) established the global stability of the optimal output process under the assumption that the shock distribution has a density whose support is an interval, bounded or unbounded. Let us conclude the paper by commenting on possible extensions.

As far as the model studied in this paper is concerned, there seems to be little room left for generalizing our first result. Extensions to more general frameworks seem possible, but may not be straightforward. The nontrivial step in such extensions is to show that the expectation of the next-period

\footnotetext{
${ }^{18}$ Their definition of $V$ corresponds to $\tilde{V}$ in our notation; see Nishimura and Stachurski (2005, Eq. (6)). Their proof actually implies (4.20).
} 
value function is continuous in current state variables (to apply the maximum theorem) and differentiable (to apply the envelope theorem).

As discussed in Section 3, there seems to be some room left for generalizing our existence result by weakening our assumptions used to prevent probability mass from escaping to infinity. But our assumptions used to prevent probability mass from escaping to zero are quite tight and possibly already the tightest. While it is straightforward to extend our existence result for general one-dimensional Markov processes to the multi-dimensional case, checking the corresponding conditions for a multi-sector growth model or a one-sector model with correlated shocks is probably not trivial, particularly when consumption is not monotone in state variables. ${ }^{19}$

Finally, it does not seem trivial to extend our stability argument for the one-sector model to cases in which the support of the shock density may not be a single interval. The main difficulty in such cases is to find a set of strictly positive measure that is covered by the support of the output density in finite time, independently of initial output. A weaker stability result, however, can be shown without requiring the shock distribution to have a density even in the case of unbounded shocks; see Zhang (2005). Like our general existence result, our stability result for general one-dimensional Markov processes can be extended to the multi-dimensional case in a straightforward manner. But it does not seem easy to extend our stability result for the one-sector model to a multi-sector model or a one-sector model with correlated shocks, particularly when, once again, consumption is not monotone in state variables.

\section{Appendix A Proof of Theorem 2.1}

Lemma A.1. $v: \mathbb{R}_{+} \rightarrow[-\infty, \infty)$ is concave, continuous, and strictly increasing. ${ }^{20}$

Proof. We show first that $v$ is strictly increasing. Let $y, \hat{y} \in \mathbb{R}_{+}$with $y<\hat{y}$. Suppose $y=0$. If $v(0)=-\infty$, then $v(\hat{y})>v(y)$ by Assumption 2.5. If

\footnotetext{
${ }^{19}$ Note that the function $V$ constructed in the proof of Theorem 3.1 consists of two monotone functions. Some of our arguments rely on the monotonicity of those functions. The monotonicity of one of them rests on the monotonicity of $c(\cdot)$.

${ }^{20}$ This lemma would be trivial if the existence of an optimal process from any initial output were known at this point.
} 
$v(0)>-\infty$, then

$$
v(\hat{y}) \geq u(\hat{y})+\beta \frac{u(0)}{1-\beta}>\frac{u(0)}{1-\beta}=v(0) .
$$

Now suppose $y>0$. Let $\left\{\left\{c_{t}^{i}, k_{t+1}^{i}\right\}\right\}_{i \in \mathbb{N}}$ be a sequence of feasible processes from $y$ such that $E \sum_{t=0}^{\infty} \beta^{t} u\left(c_{t}^{i}\right) \uparrow v(y)$ as $i \uparrow \infty$. Taking a subsequence if necessary, we may assume $c_{0}^{i} \rightarrow c_{0} \in[0, y]$. Since Assumptions 2.4 and 2.5 imply $u\left(c_{0}\right)>-\infty$, we may also assume $u\left(c_{0}^{i}\right)>-\infty$ for all $i \in \mathbb{N}$. For $i \in \mathbb{N}$, we have

$$
\begin{aligned}
v(\hat{y}) & \geq u\left(c_{0}^{i}+\hat{y}-y\right)+E \sum_{t=1}^{\infty} \beta^{t} u\left(c_{t}^{i}\right) \\
& =u\left(c_{0}^{i}+\hat{y}-y\right)-u\left(c_{0}^{i}\right)+E \sum_{t=0}^{\infty} \beta^{t} u\left(c_{t}^{i}\right) .
\end{aligned}
$$

Applying $\lim _{i \uparrow \infty}$ yields

$$
v(\hat{y}) \geq u\left(c_{0}+\hat{y}-y\right)-u\left(c_{0}\right)+v(y)>v(y) .
$$

Thus $v$ is strictly increasing.

To show the concavity of $v$, let $y, \hat{y} \in \mathbb{R}_{+}$. Let $\left\{\left\{c_{t}^{i}, k_{t+1}^{i}\right\}\right\}_{i \in \mathbb{N}}$ be as above, and define $\left\{\left\{\hat{c}_{t}^{i}, \hat{k}_{t+1}^{i}\right\}\right\}_{i \in \mathbb{N}}$ similarly. Let $\lambda \in[0,1]$. For $t \in \mathbb{Z}_{+}$and $i \in \mathbb{N}$, define $\tilde{k}_{t}^{i}=\lambda k_{t}^{i}+(1-\lambda) \hat{k}_{t}^{i}$ and $\tilde{c}_{t}^{i}=f\left(\tilde{k}_{t}^{i}, z_{t}\right)-\tilde{k}_{t+1}$. By concavity of $f(\cdot, z)$, $\lambda c_{t}^{i}+(1-\lambda) \hat{c}_{t}^{i} \leq \tilde{c}_{t}^{i}$. Thus $\left\{\tilde{c}_{t}^{i}, \tilde{k}_{t+1}^{i}\right\}$ is feasible from $\lambda y+(1-\lambda) \hat{y}$. We have

$$
\begin{aligned}
& \lambda E \sum_{t=0}^{\infty} \beta^{t} u\left(c_{t}^{i}\right)+(1-\lambda) E \sum_{t=0}^{\infty} \beta^{t} u\left(\hat{c}_{t}^{i}\right) \\
& =E \sum_{t=0}^{\infty} \beta^{t}\left[\lambda u\left(c_{t}^{i}\right)+(1-\lambda) u\left(\hat{c}_{t}^{i}\right)\right] \\
& \leq E \sum_{t=0}^{\infty} \beta^{t} u\left(\tilde{c}_{t}^{i}\right) \\
& \leq v(\lambda y+(1-\lambda) \hat{y}) .
\end{aligned}
$$

Applying $\lim _{i \uparrow \infty}$ to (A.5) yields

$$
\lambda v(y)+(1-\lambda) v(\hat{y}) \leq v(\lambda y+(1-\lambda) \hat{y}),
$$


i.e., $v$ is concave.

Since $v$ is finite on $\mathbb{R}_{++}$and concave, it is continuous except at zero. To see continuity at zero, it suffices to notice that as $y \downarrow 0$,

$$
v(y) \leq E \sum_{t=0}^{\infty} \beta^{t} u\left(\bar{c}_{t}(y)\right) \downarrow E \sum_{t=0}^{\infty} \beta^{t} u(0)=v(0),
$$

where the convergence holds by the monotone convergence theorem.

Lemma A.2. Let $y \geq 0$, and $\left\{c_{t}, k_{t+1}\right\}$ be a feasible process from $y$. Then

$$
E \sum_{i=0}^{\infty} \beta^{i} u\left(c_{1+i}\right) \leq E v\left(f\left(k_{1}, z_{1}\right)\right) .{ }^{21}
$$

Proof. Since $\left\{c_{t}\right\}$ is adapted to the filtration generated by $\left\{z_{t}\right\}_{t=1}^{\infty}$, there is a Borel function $s_{t}: \mathbb{R}_{+}^{t} \rightarrow \mathbb{R}_{+}$such that $c_{t}=s_{t}\left(z_{1}, \ldots, z_{t}\right)$ for $t \in \mathbb{N}$ (e.g., Chow and Teicher, 1997, p. 17). Let $\zeta \in \mathbb{R}_{+}$be a realization value of the random variable $z$. Define $\left\{\tilde{c}_{t}(\zeta)\right\}_{t=0}^{\infty}$ by

$$
\forall t \in \mathbb{Z}_{+}, \quad \tilde{c}_{t}(\zeta)=s_{t+1}\left(\zeta, z_{1}, \ldots, z_{t}\right) .
$$

Then it is easy to see that $\left\{\tilde{c}_{t}(\zeta)\right\}$ is feasible from $f\left(k_{1}, \zeta\right)$. Thus

$$
E \sum_{t=0}^{\infty} \beta^{t} u\left(s_{t+1}\left(\zeta, z_{1}, \ldots, z_{t}\right)\right) \leq v\left(f\left(k_{1}, \zeta\right)\right) .
$$

Because $\left\{z_{t}\right\}$ is i.i.d., it follows that

$$
E_{\left\{z_{t}\right\}_{t=2}^{\infty}} \sum_{t=0}^{\infty} \beta^{t} u\left(s_{t+1}\left(\zeta, z_{2}, \ldots, z_{t+1}\right)\right) \leq v\left(f\left(k_{1}, \zeta\right)\right),
$$

where $E_{\left\{z_{t}\right\}_{t=2}^{\infty}}$ is the expectation with respect to $\left\{z_{t}\right\}_{t=2}^{\infty}$. Replacing $\zeta$ with $z_{1}$ and applying $E_{z_{1}}$ yields (A.11).

Lemma A.3. For any feasible output process $\left\{y_{t}\right\}_{t=1}^{\infty}$ from $y_{0}>0$,

$$
\begin{gathered}
\forall t \in \mathbb{N}, \quad E v\left(y_{t}\right) \leq E \sum_{i=0}^{\infty} \beta^{i} u\left(\bar{c}_{t+i}\left(y_{0}\right)\right)_{+}<\infty, \\
\varlimsup_{t \uparrow \infty} \beta^{t} E v\left(y_{t}\right) \leq 0 .
\end{gathered}
$$

\footnotetext{
${ }^{21}$ This may appear obvious if one thinks that $\left\{c_{t}\right\}_{t=1}^{\infty}$ is feasible from $f\left(k_{1}, z_{1}\right)$. Though this is true in some sense, notice that our definition of feasibility does not require any recursive structure.
} 
Proof. Since $\bar{c}_{t}\left(y_{0}\right)$ is the maximum possible output in period $t \in \mathbb{N}$, it is easy to see that ${ }^{22}$

$$
\begin{aligned}
E v\left(y_{t}\right) & \leq E v\left(\bar{c}_{t}\left(y_{0}\right)\right) \\
& \leq E \sum_{i=0}^{\infty} \beta^{i} u\left(\bar{c}_{t+i}\left(y_{0}\right)\right) \\
& \leq E \sum_{i=0}^{\infty} \beta^{i} u\left(\bar{c}_{t+i}\left(y_{0}\right)\right)_{+}<\infty
\end{aligned}
$$

where the last inequality holds by Assumption 2.4. Hence (A.15) holds. This together with Assumption 2.4 implies (A.16).

By (2.11) and Lemma A.2, for $y \geq 0$,

$$
\begin{aligned}
v(y) & =\sup \left\{u\left(y-k_{1}\right)+\beta E \sum_{i=0}^{\infty} \beta^{i} u\left(c_{1+i}\right)\right\} \\
& \leq \sup \left\{u\left(y-k_{1}\right)+\beta E v\left(f\left(k_{1}, z_{1}\right)\right)\right\},
\end{aligned}
$$

where the suprema are taken over all feasible processes $\left\{c_{t}, k_{t+1}\right\}$ from $y$. For $x, y \geq 0$, define

$$
w(x, y)=u(y-x)+\beta E v(f(x, z)) .
$$

It follows from (A.20)-(A.22) that

$$
\forall y \geq 0, \quad v(y) \leq \sup _{x \in[0, y]} w(x, y) .
$$

The equality version of this is the Bellman equation, which is to be verified below. For $x \geq 0$, define

$$
b(x)=E v(f(x, z)) .
$$

Lemma A.4. $\forall x>0,-\infty<b(x)<\infty$, and $b: \mathbb{R}_{+} \rightarrow[-\infty, \infty)$ is concave and continuous, satisfying

$$
b_{+}^{\prime}(0)=\infty,
$$

where $b_{+}^{\prime}$ denotes the right derivative of $b$ (which exists everywhere by concavity).

\footnotetext{
${ }^{22}$ To be perfectly rigorous, (A.18) requires an argument similar to the proof of Lemma A.2.
} 
Proof. Let $x>0$. Let $y \in(0, x]$. By Assumption 2.5 and (A.23), there is $x^{\prime} \in[0, y]$ such that $w\left(x^{\prime}, y\right)>-\infty$. Hence $-\infty<b\left(x^{\prime}\right) \leq b(x)$.

To see $b(x)<\infty$, suppose $b(x)=\infty$. Let $y>x$. Let $\left\{c_{t}, k_{t+1}, y_{t+1}\right\}$ be a feasible process from $y$ with $k_{1}=x$. Then $\infty=b(x)=E v\left(f\left(k_{1}, z_{1}\right)\right)=$ $E v\left(y_{1}\right)$, contradicting (A.15).

Since $v$ and $f(\cdot, z)$ are concave and increasing, so is $b$. Thus $b$ is continuous except at zero. But continuity at zero follows by the monotone convergence theorem and Lemma A.1, so $b$ is continuous.

It remains to verify (A.25). This is trivial if $b(0)=-\infty$. Suppose $b(0)>$ $-\infty$. In this case, we may assume $u(0)=v(0)=0$ without loss of generality. For $x>0$, we have

$$
\begin{aligned}
\frac{b(x)-b(0)}{x} & =\frac{E v(f(x, z))}{x} \\
& \geq \frac{E u(f(x, z))}{x} \\
& \geq E u^{\prime}(f(x, z)) f_{1}(x, z) \uparrow \infty \quad \text { as } x \downarrow 0,
\end{aligned}
$$

where the convergence holds by the monotone convergence theorem, (2.6), and (2.7). Now (A.25) follows.

Lemma A.5. $\forall y>0$, there exists a unique $x^{*}$ with $w\left(x^{*}, y\right)=\sup _{x \in[0, y]} w(x, y)$; furthermore, $x^{*} \in(0, y)$.

Proof. Let $y>0$. By Lemma A.4, $w(\cdot, y):[0, y] \rightarrow[-\infty, \infty)$ is continuous. Thus there is $x^{*} \in[0, y]$ such that $w\left(x^{*}, y\right)=\sup _{x \in[0, y]} w(x, y)$. It is unique since $w(\cdot, y)$ is strictly concave by Lemma A.4 and strict concavity of $u$. By optimality of $x^{*}, w(x, y) \leq w\left(x^{*}, y\right)$ for $x \in[0, y]$, i.e.,

$$
\forall x \in[0, y], \quad u(y-x)+\beta b(x) \leq u\left(y-x^{*}\right)+\beta b\left(x^{*}\right) .
$$

To see $x^{*}>0$, first suppose $u(0)=-\infty$. By Lemma A.4, for $x \in$ $(0, y), w(x, y)>-\infty$; thus $x^{*}>0$ since $w(0, y)=-\infty<w(x, y)$. Now suppose $u(0)>-\infty$. If $x^{*}=y$, then $x^{*}>0$, so suppose $x^{*}<y$. Let $x \in\left(x^{*}, y\right)$. Then from (A.29),

$$
\beta \frac{b(x)-b\left(x^{*}\right)}{x-x^{*}} \leq \frac{u\left(y-x^{*}\right)-u(y-x)}{x-x^{*}} .
$$

Letting $x \downarrow x^{*}$ yields

$$
\beta b_{+}^{\prime}\left(x^{*}\right) \leq u^{\prime}\left(y-x^{*}\right)
$$


Hence $x^{*}>0$ by (A.25).

It remains to verify $x^{*}<y$. Let $x \in\left(0, x^{*}\right)$. Again from (A.29),

$$
\beta \frac{b\left(x^{*}\right)-b(x)}{x^{*}-x} \geq \frac{u(y-x)-u\left(y-x^{*}\right)}{x^{*}-x} .
$$

Letting $x \uparrow x^{*}$ yields

$$
\beta b_{-}^{\prime}\left(x^{*}\right) \geq u_{+}^{\prime}\left(y-x^{*}\right),
$$

where $b_{-}^{\prime}$ denotes the left derivative of $b$. It follows by (2.6) that $x^{*}<y$.

For $y>0$, let $k(y)=x^{*}$, where $x^{*}$ is given by Lemma A.5. Define $k(0)=0$. By Lemma A.5 and (A.23),

$$
\forall y \geq 0, \quad v(y) \leq \sup _{x \in[0, y]} w(x, y)=u(c(y))+\beta E v(f(k(y), z)) .
$$

where $c(y)=y-k(y)$ (as defined in (2.14)). Note that (A.34) holds with $y=0$, in which case both sides equal $u(0) /(1-\beta)$.

Lemma A.6. $k: \mathbb{R}_{+} \rightarrow \mathbb{R}_{+}$is continuous, and is the unique optimal policy function. Furthermore, the Bellman equation (2.12) and (2.13) hold.

Proof. Continuity follows from the maximum theorem since $w$ is continuous by Lemma A.4 and $k$ is a function rather than a correspondence. Lemma A.5 implies (2.13).

Let $y_{0} \geq 0$, and $\left\{c_{t}, k_{t+1}, y_{t+1}\right\}$ be the process generated by $k$ (or, more precisely, by (2.8) with $k$ replacing $\pi$ ). Fix $t \in \mathbb{N}$ for the moment. By (A.34),

$$
v\left(y_{t}\right) \leq u\left(c_{t}\right)+\beta E_{z_{t+1}} v\left(f\left(k\left(y_{t}\right), z_{t+1}\right)\right) .
$$

Recalling $y_{t+1}=f\left(k\left(y_{t}\right), z_{t+1}\right)$ and $c_{t}=c\left(y_{t}\right)$, applying $E_{y_{t}}$, we get

$$
E v\left(y_{t}\right) \leq E u\left(c_{t}\right)+\beta E v\left(y_{t+1}\right) .
$$


By repeated application of (A.36),

$$
\begin{aligned}
v\left(y_{0}\right) \leq & u\left(c_{0}\right)+\beta E v\left(y_{1}\right) \\
\leq & u\left(c_{0}\right)+\beta E u\left(c_{1}\right)+\beta^{2} E v\left(y_{2}\right) \\
& \vdots \\
\leq & \sum_{t=0}^{T} \beta^{t} E u\left(c_{t}\right)+\beta^{T+1} E v\left(y_{T+1}\right) \\
\leq & \sum_{t=0}^{\infty} \beta^{t} E u\left(c_{t}\right)+\varlimsup_{T \uparrow \infty} \beta^{T+1} E v\left(y_{T+1}\right) \\
\leq & E \sum_{t=0}^{\infty} \beta^{t} u\left(c_{t}\right),
\end{aligned}
$$

where the last inequality holds by (2.10) and (A.16). It follows that

$$
v\left(y_{0}\right) \leq E \sum_{t=0}^{\infty} \beta^{t} u\left(c_{t}\right) .
$$

Thus $\left\{c_{t}, k_{t+1}, y_{t+1}\right\}$ is optimal. Since it is feasible, the reverse inequality also holds, so (A.42) holds with equality. Hence (A.37), as well as (A.38)(A.41), holds with equality, i.e., $v\left(y_{0}\right)=u\left(c\left(y_{0}\right)\right)+\beta E v\left(f\left(k\left(y_{0}\right), z\right)\right.$. Since $y_{0}$ is arbitrary, the inequality in (A.34) holds with equality, i.e., the Bellman equation (2.12) holds.

Since (A.37)-(A.41) hold for any $y_{0} \geq 0, k$ is an optimal policy function. For uniqueness, let $\tilde{k}$ be a feasible policy function with $\tilde{k}\left(y_{0}\right) \neq k\left(y_{0}\right)$ for some $y_{0}>0$. Let $\left\{\tilde{c}_{t}, \tilde{k}_{t+1}, \tilde{y}_{t+1}\right\}$ be the process generated by $\tilde{k}$ from $y_{0}$. By the Bellman equation (2.12) and the argument leading to (A.36),

$$
\forall t \in \mathbb{Z}_{+}, \quad \operatorname{Ev}\left(\tilde{y}_{t}\right) \geq \operatorname{Eu}\left(\tilde{c}_{t}\right)+\beta E v\left(\tilde{y}_{t+1}\right) .
$$

Hence all the inequalities in (A.37)-(A.41) are reversed (with $\tilde{c}_{t}$ and $\tilde{y}_{t}$ replacing $c_{t}$ and $y_{t}$ ), and the reversed version of (A.37) holds strictly by Lemma A.5 since $\tilde{k}\left(y_{0}\right) \neq k\left(y_{0}\right)$. Thus $E \sum_{t=0}^{\infty} \beta u\left(\tilde{c}_{t}\right)<v\left(y_{0}\right)$, i.e., $\tilde{k}$ is not optimal. It follows that $k$ is the unique optimal policy function.

Lemma A.7. $v$ is continuously differentiable on $\mathbb{R}_{++}$, and the Euler equation (2.15) holds. 
Proof. It suffices to show that $b$ as defined in (A.24) is differentiable on $\mathbb{R}_{++}$ and

$$
\forall x>0, \quad b^{\prime}(x)=E u^{\prime}(c(f(x, z))) f_{1}(x, z) .
$$

For then the Euler equation (2.15) is simply the first order condition for the maximization problem on the right-hand side of (2.12).

To verify (A.44), applying the envelope theorem (e.g., Milgrom and Segal, 2002, Corollary 3) to the Bellman equation (2.12), we find that $v$ is differentiable on $\mathbb{R}_{++}$and

$$
\forall y>0, \quad v^{\prime}(y)=u^{\prime}(c(y))
$$

Thus $v$ is differentiable on $\mathbb{R}_{++}$. Since $k$ is continuous by Lemma A.6, so is $c$ as well as $v^{\prime}$.

Let $x>0$. Recalling Lemma A.4, we see that for $h>0$,

$$
\frac{b(x+h)-b(x)}{h}=E \frac{v(f(x+h, z))-v(f(x, z))}{h} .
$$

By concavity, $[v(f(x+h, z))-v(f(x, z))] / h$ increases as $h$ decreases. Thus by the monotone convergence theorem, (A.45), and the chain rule,

$$
b_{+}^{\prime}(x)=E u^{\prime}(c(f(x, z))) f_{1}(x, z) .
$$

Let $\bar{h} \in(0, x)$. For $h \in(0, \bar{h}]$,

$$
\begin{aligned}
\frac{b(x)-b(x-h)}{h} & =E \frac{v(f(x, z))-v(f(x-h, z))}{h} \\
& \leq E \frac{v(f(x, z))-v(f(x-\bar{h}, z))}{\bar{h}}<\infty,
\end{aligned}
$$

where the first inequality holds since $[v(f(x, z))-v(f(x-h, z))] / h$ decreases as $h$ decreases. Thus $b_{-}^{\prime}(x)$ equals the right-hand side of (A.47) again by the monotone convergence theorem, (A.45), and the chain rule. Now (A.44) follows.

Lemma A.8. $v$ is strictly concave, and $k$ and $c$ are strictly increasing. ${ }^{23}$

\footnotetext{
${ }^{23}$ This is a well known result in the case that $f(\cdot, z)$ is strictly concave (e.g., Brock and Mirman, 1972, Lemma 1.1). Note that $f(\cdot, z)$ is only assumed to be concave here.
} 
Proof. Let $y \geq 0$ and $\hat{y}>y$. If $y=0$, then $k(y)<k(\hat{y})$ by Lemma A.5. Suppose $y>0$. If $k(y)=k(\hat{y})$, then by the Euler equation $(2.15), u^{\prime}(y-$ $k(y))=u^{\prime}(\hat{y}-k(y))$, a contradiction. Hence $k$ is strictly increasing.

It follows from (A.45) and concavity of $v$ that $c$ is increasing. To conclude that $c$ is strictly increasing, it suffices to show that $v$ is strictly concave. Let $y, \hat{y} \geq 0$ with $y \neq \hat{y}$. Then (A.5)-(A.8) hold even if $\left\{c_{t}^{i}\right\}$ and $\left\{\hat{c}_{t}^{i}\right\}$ are optimal consumption processes from $y$ and $\hat{y}$. Since $v$ is strictly increasing, $P\left(c_{t}^{i} \neq \hat{c}_{t}^{i}\right)>0$ for some $t \in \mathbb{Z}_{+}$. Thus by strict concavity of $u$ and Jensen's inequality, (A.7) holds strictly. It follows that $v$ is strictly concave.

Now Lemmas A.1 and A.6-A.8 establish Theorem 2.1.

\section{Appendix B Proofs of Section 3 Results}

\section{B.1 Proof of Lemma 3.1}

Since $V(0)=\infty$, by $(3.16)$,

$$
\forall y>0, \quad P(m(y, z)=0)=0 .
$$

Hence (3.11) holds, so we may restrict the state space of $\left\{y_{t}\right\}$ to $\mathbb{R}_{++}$provided $y_{0}>0$. Let $y_{0}>0$. By (3.16), $E_{z_{t+1}}\left[V\left(m\left(y_{t}, z_{t+1}\right)\right)\right] \leq \lambda V\left(y_{t}\right)+\alpha$ for $t \in \mathbb{Z}_{+}$. Applying $E_{y_{t}}$, we get

$$
\begin{aligned}
E V\left(y_{t+1}\right) & \leq \lambda E V\left(y_{t}\right)+\alpha \\
& \leq \lambda^{t+1} V\left(y_{0}\right)+\left(1+\lambda+\cdots+\lambda^{t}\right) \alpha,
\end{aligned}
$$

where the second inequality holds for $t \geq 1$. It follows that $\varlimsup_{t \uparrow \infty} E V\left(y_{t}\right) \leq$ $\alpha /(1-\lambda)$. Since the Markov process (3.2) has the Feller property by Stokey and Lucas (1989, Exercise 8.10), the conclusion follows by Meyn and Tweedie (1996, Proposition 12.1.3).

\section{B.2 Proof of Theorem 3.1}

Lemma B.1. Let $\zeta$ be a positive random variable such that

$$
\exists \hat{r}>0, \quad E \zeta^{\hat{r}}<\infty .
$$


Then

$$
\begin{gathered}
\forall r \in(0, \hat{r}], \quad E \ln \zeta \leq \ln \left[E \zeta^{r}\right]^{\frac{1}{r}}, \\
\lim _{r \downarrow 0} \ln \left[E \zeta^{r}\right]^{\frac{1}{r}}=E \ln \zeta .
\end{gathered}
$$

Proof. See Hardy et al. (1952, pp. 137-139).

Lemma B.2. There exist $r, \tilde{y}>0$ such that

$$
\left[E\left\{\beta f_{1}(k(\tilde{y}), z)\right\}^{-r}\right]^{\frac{1}{r}}<1 .
$$

Proof. Let $\tilde{r}, \tilde{x}>0$ be as in Assumption 3.4. By concavity,

$$
\forall x \in(0, \tilde{x}], \quad E f_{1}(x, z)^{-\tilde{r}}<\infty .
$$

By Assumption 3.5, there is $\tilde{y}>0$ such that

$$
\text { (i) } E \ln \left[\beta f_{1}(k(\tilde{y}), z)\right]>0, \quad \text { (ii) } k(\tilde{y}) \leq \tilde{x} .
$$

By (B.9)(ii), (B.8), and Lemma B.1 with $\left.x=\left[\beta f_{1}(k(\tilde{y}), z)\right)\right]^{-1}$,

$$
\lim _{r \downarrow 0} \ln \left[E\left\{\beta f_{1}(k(\tilde{y}), z)\right\}^{-r}\right]^{\frac{1}{r}}=-E \ln \left\{\beta f_{1}(k(\tilde{y}), z)\right\}<0,
$$

where the inequality holds by (B.9)(i). It follows that for small enough $r>0, \ln \left[E\left\{\beta f_{1}(k(\tilde{y}), z)\right\}^{-r}\right]^{\frac{1}{r}}<0$, which is equivalent to (B.7).

Lemma B.3. Let $r, \tilde{y}>0$ satisfy (B.7). For $y>0$, define

$$
w_{1}(y)=u^{\prime}(c(y))^{\frac{r}{1+r}} .
$$

Then there exist $\lambda_{1} \in(0,1)$ and $\alpha_{1}>0$ such that

$$
\forall y>0, \quad E w_{1}(f(k(y), z)) \leq \lambda_{1} w_{1}(y)+\alpha_{1} .
$$

Proof. Let $p=(1+r) / r$ and $q=1+r$. Note that

$$
\text { (i) } p, q \in(1, \infty), \quad \text { (ii) } \frac{1}{p}+\frac{1}{q}=1, \quad \text { (iii) } \frac{q}{p}=r .
$$

By (B.7),

$$
\begin{gathered}
\lambda_{1} \equiv\left[E\left\{\beta f_{1}(k(\tilde{y}), z)\right\}^{-r}\right]^{\frac{1}{r} \frac{1}{p}}<1, \\
\forall y \in(0, \tilde{y}], \quad\left[E\left\{\beta f_{1}(k(y), z)\right\}^{-r}\right]^{\frac{1}{r}} \frac{1}{p} \leq \lambda_{1},
\end{gathered}
$$


where the second inequality holds since $f_{1}(\cdot, z)$ is decreasing and $k$ is increasing.

For $y \in(0, \tilde{y}]$ and $c^{\prime}=c(f(k(y), z))$, by (B.15), the Euler equation (2.15), (B.13)(iii), and Hölder's inequality, ${ }^{24}$

$$
\begin{aligned}
& u^{\prime}(c(y))^{\frac{1}{p}}\left[E\left\{\beta f_{1}(k(y), z)\right\}^{-r}\right]^{\frac{1}{r} \frac{1}{p}} \\
& =\left[E\left\{\beta u^{\prime}\left(c^{\prime}\right) f_{1}(k(y), z)\right\}\right]^{\frac{1}{p}}\left[E\left\{\beta f_{1}(k(y), z)\right\}^{-\frac{q}{p}}\right]^{\frac{1}{q}} \\
& \geq E\left[\left\{\beta u^{\prime}\left(c^{\prime}\right) f_{1}(k(y), z)\right\}^{\frac{1}{p}}\left\{\beta f_{1}(k(y), z)\right\}^{-\frac{1}{p}}\right]=E u^{\prime}\left(c^{\prime}\right)^{\frac{1}{p}} .
\end{aligned}
$$

Recalling the definition of $w_{1}$, by (B.16)-(B.18) and (B.15), we have

$$
\forall y \in(0, \tilde{y}], \quad E w_{1}(f(k(y), z)) \leq \lambda_{1} w_{1}(y) .
$$

Since $k$ and $c$ are increasing,

$$
\begin{aligned}
\forall y \geq \tilde{y}, \quad E w_{1}(f(k(y), z)) & =E u^{\prime}(c(f(k(y), z)))^{\frac{1}{p}} \\
& \leq E u^{\prime}(c(f(k(\tilde{y}), z)))^{\frac{1}{p}} \equiv \alpha_{1} .
\end{aligned}
$$

Now (B.12) follows from (B.19)-(B.21).

Lemma B.4. There exist $\lambda_{2} \in(0,1)$ and $\alpha_{2}>0$ such that

$$
\forall y>0, \quad E f(k(y), z) \leq \lambda_{2} y+\alpha_{2} .
$$

Proof. By (3.9), concavity, and the monotone convergence theorem,

$$
\begin{aligned}
\lim _{y \uparrow \infty} E \frac{f(y, z)}{y} & =E \lim _{y \uparrow \infty} \frac{f(y, z)}{y} \\
& =E \lim _{y \uparrow \infty} f_{1}(y, z) \equiv \underline{\lambda}<1,
\end{aligned}
$$

where the inequality holds by Assumption 3.3 and the monotone convergence theorem. Let

$$
\lambda_{2} \in(\underline{\lambda}, 1) .
$$

By (B.23) and (B.24), there is $\hat{y}>0$ such that

$$
\forall y \geq \hat{y}, \quad E \frac{f(y, z)}{y} \leq \lambda_{2} .
$$

\footnotetext{
${ }^{24}$ If $p$ and $q$ satisfy (B.13)(i) and (B.13)(ii), then $E|\xi \eta| \leq\left(E|\xi|^{p}\right)^{\frac{1}{p}}\left(E|\eta|^{q}\right)^{\frac{1}{q}}$ for random variables $\xi$ and $\eta$ with $E|\xi|^{p}, E|\eta|^{q}<\infty$ (e.g., Dudley, 2002, p. 154). In our case, $\xi=$ $\left\{\beta u^{\prime}\left(c^{\prime}\right) f_{1}(k(y), z)\right\}^{\frac{1}{p}}$ and $\eta=\left\{\beta f_{1}(k(y), z)\right\}^{-\frac{1}{p}}$.
} 
Since $k(y) \leq y$ for all $y \geq 0, E f(k(y), z) \leq E f(y, z)$. This together with (B.26) shows

$$
\forall y \geq \hat{y}, \quad E f(k(y), z) \leq \lambda_{2} y
$$

Since $k$ is increasing,

$$
\forall y \in(0, \hat{y}], \quad \operatorname{Ef}(k(y), z) \leq \operatorname{Ef}(k(\hat{y}), z) \equiv \alpha_{2} .
$$

Now (B.22) follows from (B.27) and (B.28).

To complete the proof of Theorem 3.1, define

$$
\forall y>0, \quad V(y)=w_{1}(y)+y,
$$

where $w_{1}$ is given by (B.11). Define $V(0)=\infty$. Then (3.14) holds. Let $\lambda=\max \left\{\lambda_{1}, \lambda_{2}\right\}$ and $\alpha=\alpha_{1}+\alpha_{2}$. Then (3.15) and (3.16) follow from (B.12) and (B.22). Since $m$ as defined in (3.1) is continuous, an invariant distribution exists by Lemma 3.1.

\section{Appendix C Proofs of Section 4 Results}

\section{C.1 Proof of Lemma 4.1}

We apply Meyn and Tweedie (MT) (1996, Theorem 16.1.2) to show that the Markov process (3.2), or interchangeably the operator $T$ defined by (4.13), is $V$-uniformly ergodic. This implies (4.20) by MT (Theorem 16.0.1). Hence by MT (Theorem 16.1.2), the lemma holds if $T$ is $\psi$-irreducible and aperiodic and if (V4) in MT (Chapter 15) holds with some petite set $C \subset \mathbb{R}_{++}$and some function $\tilde{V}: \mathbb{R}_{++} \rightarrow[1, \infty)$.

$\psi$-Irreducibility: For any $B \in \mathcal{B}$, the mapping $y \mapsto \int_{B} \underline{q}\left(y, y^{\prime}\right) d y^{\prime}$ is lower semicontinuous by Fatou's lemma and lower semicontinuity of $q$. Thus $T$ is a T-chain (MT, Chapter 6) by (4.16) and (4.17). For $B \in \mathcal{B}$, define $\varphi(B)=\int 1_{A \cap B}(y) d y .{ }^{25}$ Then by (4.19) and MT (Proposition 4.2.1(ii)), $T$ is $\varphi$-irreducible. By MT (Proposition 4.2.2), $T$ is $\psi$-irreducible for some maximal irreducibility measure $\psi$.

\footnotetext{
${ }^{25}$ For $W \in \mathcal{B}, 1_{W}(\cdot)$ is the indicator function on $W$; i.e., $1_{W}(y)=1$ if $y \in W$ and $1_{W}(y)=0$ otherwise.
} 
Aperiodicity: It suffices to show that $T$ is strongly aperiodic (MT, Chapter 5). By (4.18) and lower semicontinuity of $\underline{q}$, there is $\epsilon>0$ such that

$$
\forall y, y^{\prime} \in C \equiv[\tilde{y}-\epsilon, \tilde{y}+\epsilon], \quad \underline{q}\left(y, y^{\prime}\right)>0 .
$$

We verify that $\nu(C)>0$ and $C$ is $\nu$-small with $\nu$ defined below. Let $\theta=$ $\min _{y, y^{\prime} \in C} \underline{q}\left(y, y^{\prime}\right)>0$. For $B \subset \mathcal{B}$, define

$$
\nu(B)=\theta \int 1_{B \cap C}(x) d x
$$

Then $\nu(C)=\theta \int 1_{C}(x) d x=2 \theta \epsilon>0$. For $y \in C$ and $B \in \mathcal{B}$,

$$
\begin{aligned}
\int_{B} q\left(y, y^{\prime}\right) d y^{\prime} & \geq \int \underline{q}\left(y, y^{\prime}\right) 1_{B \cap C}\left(y^{\prime}\right) d y^{\prime} \\
& \geq \theta \int 1_{B \cap C}\left(y^{\prime}\right) d y^{\prime}=\nu(B) .
\end{aligned}
$$

Hence $C$ is $\nu$-small. It follows that $T$ is strongly aperiodic.

$(V 4)$ : Define

$$
\tilde{V}=V+1, \quad \tilde{\alpha}=\alpha+(1-\lambda) .
$$

Then (3.15) and (3.16) still hold with $\tilde{V}$ and $\tilde{\alpha}$ replacing $V$ and $\alpha$, and $\tilde{V}: \mathbb{R}_{++} \rightarrow[1, \infty) \cdot{ }^{26}$ Let $\eta=(1-\lambda) / 2$. Let

$$
\tilde{C}=\{y>0 \mid \tilde{V}(y) \leq \tilde{\alpha} / \eta\} .
$$

By (3.15), $\tilde{C}$ is bounded and bounded away from zero. Hence there is a compact interval $C \subset \mathbb{R}_{++}$with $\tilde{C} \subset C$ (C here is different from $C$ in (C.1)). Since $T$ is a T-chain, $C$ is petite by MT (Theorem 6.2.5). From (3.16),

$$
\begin{aligned}
E[\tilde{V}(m(y, z))]-\tilde{V}(y) & \leq-(1-\lambda) \tilde{V}(y)+\tilde{\alpha} \\
& =-\eta \tilde{V}(y)+\tilde{\alpha}-\eta \tilde{V}(y) \\
& \leq-\eta \tilde{V}(y)+\tilde{\alpha} 1_{C}(y) .
\end{aligned}
$$

Hence (V4) in MT (Chapter 15) holds with the petite set $C$.

\footnotetext{
${ }^{26}$ The following argument is adapted from MT (Lemma 15.2.8).
} 


\section{C.2 Proof of Theorem 4.1}

Let $\gamma>0$. Define $\underline{q}: \mathbb{R}_{++}^{2} \rightarrow \mathbb{R}_{+}$by

$$
\underline{q}\left(y, y^{\prime}\right)= \begin{cases}\min \left\{\frac{\underline{\phi}\left(\zeta\left(y, y^{\prime}\right)\right)}{f_{2}\left(k(y), \zeta\left(y, y^{\prime}\right)\right)}, \gamma\right\} & \text { if } y^{\prime} \in(\underline{h}(y), \bar{h}(y)), \\ 0 & \text { otherwise, }\end{cases}
$$

where $\phi$ is given by Assumption 4.1. Note that for $y, y^{\prime}>0$,

$$
\underline{q}\left(y, y^{\prime}\right)>0 \Leftrightarrow q\left(y, y^{\prime}\right)>0 \Leftrightarrow y^{\prime} \in(\underline{h}(y), \bar{h}(y)) .
$$

It is easy to see that

$$
\underline{q} \text { is continuous and satisfies (4.16) and (4.17). }
$$

Let $L^{1}$ be the set of Borel functions $g: \mathbb{R}_{++} \rightarrow \mathbb{R}_{+}$with $\int g(y) d y<\infty$. Define $\underline{T}: L^{1} \rightarrow L^{1}$ by

$$
\forall y^{\prime}>0, \quad(\underline{T g})\left(y^{\prime}\right)=\int \underline{q}\left(y, y^{\prime}\right) g(y) d y .
$$

Note from (4.16) and (C.12) that

$$
\forall g \in L^{1}, \quad \underline{T g} \leq T g .
$$

Lemma C.1. $\forall g \in L^{1}, \underline{T g}$ is continuous.

Proof. Fix $y^{\prime}>0$. For $\epsilon \in \mathbb{R}$ with $y^{\prime}+\epsilon>0$, we have

$$
\left|(\underline{T} g)\left(y^{\prime}+\epsilon\right)-(\underline{T} g)\left(y^{\prime}\right)\right| \leq \int\left|\underline{q}\left(y, y^{\prime}+\epsilon\right)-\underline{q}\left(y, y^{\prime}\right)\right| g(y) d y .
$$

By (C.10), $\left|q\left(y, y^{\prime}+\epsilon\right)-q\left(y, y^{\prime}\right)\right| g(y) \leq \gamma g(y)$. Since $g$ is integrable and $q$ is continuous, applying the dominated convergence theorem to (C.15) yields

$$
\begin{aligned}
& \lim _{\epsilon \rightarrow 0}\left|(\underline{T} g)\left(y^{\prime}+\epsilon\right)-(\underline{T} g)\left(y^{\prime}\right)\right| \\
& \leq \int \lim _{\epsilon \rightarrow 0}\left|\underline{q}\left(y, y^{\prime}+\epsilon\right)-\underline{q}\left(y, y^{\prime}\right)\right| g(y) d y=0 .
\end{aligned}
$$

Hence $\underline{T g}$ is continuous. 
Lemma C.2. $\forall g \in D$, there exist $a, b>0$ with $a<b$ such that $(a, b) \subset$ $S(T g)$.

Proof. Since $T g \in D$, there is $y^{\prime}>0$ such that $(T g)\left(y^{\prime}\right)>0$. By (C.11), $(\underline{T g})\left(y^{\prime}\right)>0$. Since $\underline{T g}$ is continuous by Lemma C.1, $\underline{T g}>0$ on some open interval $(a, b) \subset \mathbb{R}_{++}$containing $y^{\prime}$. Hence $(a, b) \in S(T g)$ by (C.14).

Note from Assumption 4.2 that

$$
\bar{z}=\infty \quad \Rightarrow \quad \forall y>0, \bar{h}(y)=\infty .
$$

If $\bar{z}=\infty$, we extend the domain of $\bar{h}$ to $(0, \infty]$ by defining

$$
\bar{z}=\infty \quad \Rightarrow \quad \bar{h}(\infty)=\infty .
$$

Lemma C.3. Let $g \in D, a \geq 0$, and $b \in(a, \infty]$. Then

$$
\text { (i) }(a, b) \subset S(g) \quad \Rightarrow \quad \text { (ii) }(\underline{h}(a), \bar{h}(b)) \subset S(T g) \text {. }
$$

Proof. Assume (i). Let $y^{\prime} \in(\underline{h}(a), \bar{h}(b))$. Since $\underline{h}$ and $\bar{h}$ are continuous and increasing,

$$
(\underline{h}(a), \bar{h}(b))=\cup_{y \in(a, b)}(\underline{h}(y), \bar{h}(y)) .
$$

Hence there is $\tilde{y} \in(a, b)$ with $y^{\prime} \in(\underline{h}(\tilde{y}), \bar{h}(\tilde{y}))$. By (C.11), $\underline{q}\left(\tilde{y}, y^{\prime}\right)>0$. By continuity, there is a nonempty open interval $(\tilde{a}, \tilde{b}) \subset(a, b)$ such that $\underline{q}\left(y, y^{\prime}\right)>0$ for $y \in(\tilde{a}, \tilde{b})$. We have $\underline{q}\left(y, y^{\prime}\right) g(y)>0$ for $y \in(\tilde{a}, \tilde{b}) \subset(a, b)$, so

$$
(T g)\left(y^{\prime}\right) \geq(\underline{T} g)\left(y^{\prime}\right) \geq \int_{\tilde{a}}^{\tilde{b}} \underline{q}\left(y, y^{\prime}\right) g(y) d y>0 .
$$

Hence $y^{\prime} \in S(T g)$.

Lemma C.4. Let $y>0$. (i) If $\beta E f_{1}(k(y), z) \geq 1$, then $\bar{h}(y)>y$. (ii) If $\beta E f_{1}(k(y), z) \leq 1$, then $\underline{h}(y)<y$.

Proof. To see (i), suppose $\bar{h}(y) \leq y$. Recall the Euler equation (2.15):

$$
\begin{aligned}
u^{\prime}(c(y)) & =\beta E\left[u^{\prime}(c(f(k(y), z))) f_{1}(k(y), z)\right] \\
& >\beta E\left[u^{\prime}(c(y)) f_{1}(k(y), z)\right],
\end{aligned}
$$

where the inequality holds since $f(k(y), z)<\bar{h}(y) \leq y$ with probability 1 . It follows that $1>\beta E f_{1}(k(y), z)$. This establishes (i). The proof of (ii) is similar. 
Lemma C.5. (i) For sufficiently small $y>0, \bar{h}(y)>y$. (ii) For sufficiently large $y>0, \underline{h}(y)<y$.

Proof. Part (ii) is immediate from Lemma C.4(ii) and Assumption 3.3. To see (i), note from Assumption 3.5 that $E \ln \left[\beta f_{1}(k(y), z)\right]>0$ for small enough $y>0$. For such $y$, by Jensen's inequality,

$$
0<E \ln \left[\beta f_{1}(k(y), z)\right] \leq \ln E\left[\beta f_{1}(k(y), z)\right] .
$$

Hence $\beta E f_{1}(k(y), z)>1$, so $\bar{h}(y)>y$ by Lemma C.4(i).

Define

$$
\begin{aligned}
& \underline{y}=\max \{y \geq 0 \mid y=\underline{h}(y)\}, \\
& \bar{y}= \begin{cases}\min \{y>0 \mid y=\bar{h}(y)\} & \text { if } \exists y>0, y=\bar{h}(y), \\
\infty & \text { otherwise. }\end{cases}
\end{aligned}
$$

By Lemma C.5,

$$
\underline{y}<\infty, \quad \bar{y}>0 .
$$

Lemma C.6. $\underline{y}<\bar{y}$.

Proof. By (C.28), $\underline{y}<\bar{y}$ if $\underline{y}=0$ or $\bar{y}=\infty$. Suppose $\underline{y}>0$ and $\bar{y}<\infty .{ }^{27}$ Since $f(k(\underline{y}), z)>\underline{y}$ and $f(\bar{k}(\bar{y}), z)<\bar{y}$ with probability 1, from the Euler equation $(2.15)$,

$$
\begin{aligned}
& u^{\prime}(c(\underline{y}))<\beta E\left[u^{\prime}(c(\underline{y})) f_{1}(k(\underline{y}), z)\right], \\
& u^{\prime}(c(\bar{y}))>\beta E\left[u^{\prime}(c(\bar{y})) f_{1}(k(\bar{y}), z)\right] .
\end{aligned}
$$

Hence

$$
\beta E f_{1}(k(\bar{y}), z)<1<\beta E f_{1}(k(\underline{y}), z) .
$$

Thus $\underline{y}<\bar{y}$ again.

Lemma C.7. There exist $\tilde{a}, \tilde{b}>0$ with $\tilde{a}<\tilde{b}$ such that $A \equiv(\tilde{a}, \tilde{b})$ satisfies (4.19).

\footnotetext{
${ }^{27}$ The following argument is a simplified version of that of Brock and Mirman (1972, Lemma 3.4).
} 
Proof. Let $\tilde{a}, \tilde{b}>0$ be such that $y<\tilde{a}<\tilde{b}<\bar{y}$. Let $A=(\tilde{a}, \tilde{b})$. Let $y_{0}>0$, and $g_{t}=g_{t}^{y_{0}}$ for $t \in \mathbb{N}$ for simplicity. Since $g_{1} \in D$, by Lemma C.2 there is an open interval $\left(a_{2}, b_{2}\right) \subset S\left(g_{2}\right)=S\left(T g_{1}\right)$. For $t \geq 2$, define $a_{t+1}=\underline{h}\left(a_{t}\right)$ and $b_{t+1}=\bar{h}\left(b_{t}\right)$. By Lemma C.5(ii), $\lim \sup _{t \uparrow \infty} a_{t}<\infty$. Since $\underline{h}$ is continuous and increasing, $\lim _{t \uparrow \infty} a_{t} \leq \underline{y}$. By Lemma C.5(i), $\liminf _{t \uparrow \infty} b_{t}>0$. If $\bar{z}<\infty$, since $\bar{h}$ is continuous and increasing, $\lim _{t \uparrow \infty} b_{t} \geq \bar{y}$. This inequality is trivial if $\bar{z}=\infty$, in which case $\bar{y}=\infty$ and $b_{t}=\infty$ for $t \geq 2$. It follows that $A \subset S\left(g_{t}\right)$ for $t$ large enough, which implies (4.19).

Let us now complete the proof of Theorem 4.1 by applying Lemma 4.1. Hypothesis (i) follows from the proof of Theorem 3.1; see (B.29). Equation (4.9) is a property of the model, so (ii) holds. Let $\tilde{y} \in(y, \bar{y})$. Then $\underline{h}(\tilde{y})<$ $\tilde{y}<\bar{h}(\tilde{y})$, so $q(\tilde{y}, \tilde{y})>0$ by (C.11). This and (C.12) show (iii). Hypothesis (iv) is immediate from Lemma C.7. Now Theorem 4.1 follows from Lemma 4.1 .

\section{References}

Bhattacharaya, R.N., Majumdar M., 1989. Controlled semi-Markov models - the discounted case. Journal of Statistical Planning and Inference 21, $365-381$.

Brock, W.A., Mirman, L.J., 1972. Optimal economic growth and uncertainty: the discounted case. Journal of Economic Theory 4, 479-513.

Chow, Y.S., Teicher, H., 1997. Probability Theory: Independence, Interchangeability, and Martingales, 3rd ed. Springer, New York.

Dudley, R.M, 2002. Real Analysis and Probability. Cambridge University Press, Cambridge.

Durán, J., 2003. Discounting long run average growth in stochastic dynamic programs. Economic Theory 22, 395-413.

Hardy, G., Littlewood, J.E., Pólya, G., 1952. Inequalities, 2nd ed. Cambridge University Press, Cambridge.

Hernández-Lerma, O., Lasserre, J.B., 1996. Discrete-Time Markov Control Processes: Basic Optimality Criteria. Springer, New York.

Hernández-Lerma, O., Lasserre, J.B., 1999. Further Topics on Discrete-Time Markov Control Processes. Springer, New York. 
Kamihigashi, T., 2005. Almost sure convergence to zero in stochastic growth models. Economic Theory, forthcoming.

Kamihigashi, T., Roy, S., 2005. A nonsmooth, nonconvex model of optimal growth. Journal of Economic Theory, forthcoming.

Le Van, C., Morhaim, L., 2002. Optimal growth models with bounded or unbounded returns: a unifying approach. Journal of Economic Theory 105, $158-187$.

Meyn, S.P., Tweedie, R.L., 1996. Markov Processes and Stochastic Stability. Springer-Verlag, Berlin.

Milgrom, P., Segal, I., 2002. Envelope theorems for arbitrary choice sets. Econometrica $70,583-601$.

Mitra, T., Montrucchio, L., Prvileggi, F., 2004. The nature of the steady state in models of optimal growth under uncertainty. Economic Theory 23, $39-71$.

Mitra, T., Roy, S., 2005. On the possibility of extinction in a class of Markov processes in economics. Department of Economics, Southern Methodist University.

Mitra, T., Roy, S., 2006. Optimal exploitation of renewable resources under uncertainty and the extinction of species. Economic Theory 28, 1-23.

Mirman, L.J., Zilcha, I., 1975. On optimal growth under uncertainty. Journal of Economic Theory 11, 329-339.

Nishimura, K., Rudnicki, R., Stachurski, J., 2006. Stochastic optimal growth with nonconvexitites. Journal of Mathematical Economics 42, 74-96.

Nishimura, K,. Stachurski, J., 2005. Stability of stochastic optimal growth models: a new approach. Journal of Economic Theory 122, 100-118.

Olson, L.J., Roy, S., 2005. Theory of stochastic optimal economic growth. In: C. Le Van, Dana, R.A., Mitra, T., Nishimura, K. (Eds), Handbook of Optimal Growth, Vol. 1: The Discrete Time Horizon. Springer, New York, forthcoming.

Schäl, M., 1975. Conditions for optimality in dynamic programming and for the limit of $n$-stage optimal policies to be optimal. Z. Wahrscheinlichkeitstheorie verw. Gebiete 32, 179-196.

Stachurski, J., 2002. Stochastic optimal growth with unbounded shock. Journal of Economic Theory 106, 40-65. 
Stokey, N., Lucas, R.E., Jr., 1989. Recursive Methods in Economic Dynamics. Harvard University Press, Cambridge, MA.

Zhang, Y., 2005. Stochastic optimal growth with non-compact state space. Department of Economics, University of Minnesota. 